\title{
INITIAL BOUNDARY VALUE PROBLEM FOR A SEMILINEAR PARABOLIC EQUATION WITH ABSORPTION AND NONLINEAR NONLOCAL BOUNDARY CONDITION
}

\author{
ALEXANDER GLADKOV
}

\begin{abstract}
In this paper we consider an initial boundary value problem for a semilinear parabolic equation with absorption and nonlinear nonlocal Neumann boundary condition. We prove comparison principle, the existence theorem of a local solution and study the problem of uniqueness and nonuniqueness.
\end{abstract}

\section{INTRODUCTION}

In this paper we consider the initial boundary value problem for the following semilinear parabolic equation

$$
u_{t}=\Delta u-c(x, t) u^{p}, x \in \Omega, t>0,
$$

with nonlinear nonlocal boundary condition

$$
\frac{\partial u(x, t)}{\partial \nu}=\int_{\Omega} k(x, y, t) u^{l}(y, t) d y, x \in \partial \Omega, t \geq 0,
$$

and initial datum

$$
u(x, 0)=u_{0}(x), x \in \Omega,
$$

where $p>0, l>0, \Omega$ is a bounded domain in $\mathbb{R}^{n}$ for $n \geq 1$ with smooth boundary $\partial \Omega, \nu$ is unit outward normal on $\partial \Omega$.

Throughout this paper we suppose that the functions $c(x, t), k(x, y, t)$ and $u_{0}(x)$ satisfy the following conditions:

$$
\begin{gathered}
c(x, t) \in C_{l o c}^{\alpha}(\bar{\Omega} \times[0,+\infty)), 0<\alpha<1, c(x, t) \geq 0 \\
k(x, y, t) \in C(\partial \Omega \times \bar{\Omega} \times[0,+\infty)), k(x, y, t) \geq 0 \\
u_{0}(x) \in C^{1}(\bar{\Omega}), u_{0}(x) \geq 0 \text { in } \Omega, \frac{\partial u_{0}(x)}{\partial \nu}=\int_{\Omega} k(x, y, 0) u_{0}^{l}(y) d y \text { on } \partial \Omega .
\end{gathered}
$$

A lot of articles have been devoted to the investigation of initial boundary value problems for parabolic equations and systems with nonlinear nonlocal Dirichlet boundary condition (see, for example, [6, 8, 12, 13, 14, 17, 18, 19, 23, 24, 26, 27. and the references therein). In particular, the initial boundary value problem for equation (1.1) with nonlocal boundary condition

$$
u(x, t)=\int_{\Omega} k(x, y, t) u^{l}(y, t) d y, x \in \partial \Omega, t>0,
$$

was considered for $c(x, t) \leq 0$ and $c(x, t) \geq 0$ in [13, 14] and [17, 18, respectively. The problem (1.1)-(1.3) with $c(x, t) \leq 0$ were investigated in [15, 16].

2010 Mathematics Subject Classification. Primary 35K20, 35K58, 35K61.

Key words and phrases. semilinear parabolic equation, nonlocal boundary condition, local solution, uniqueness. 
We note that for $p<1$ and $l<1$ the nonlinearities in equation (1.1) and boundary condition (1.2) are non-Lipschitzian. The problem of uniqueness and nonuniqueness for different parabolic nonlinear equations with non-Lipschitzian data in bounded domain has been addressed by several authors (see, for example, 2, 4, 5, 7, 9, 14, 18, 21] and the references therein).

The aim of this paper is to study problem (1.1)-(1.3) for any $p>0$ and $l>0$. We prove existence of a local solution and establish some uniqueness and nonuniqueness results.

This paper is organized as follows. In the next section we prove the existence of a local solution. Comparison principle and the problem of uniqueness and nonuniqueness for (1.1)-(1.3) are investigated in Section 3 .

\section{LOCAL EXISTENCE}

In this section a local existence theorem for (1.1)-(1.3) will be proved. We begin with definitions of a supersolution, a subsolution and a maximal solution of (1.1)(1.3). Let $Q_{T}=\Omega \times(0, T), S_{T}=\partial \Omega \times(0, T), \Gamma_{T}=S_{T} \cup \bar{\Omega} \times\{0\}, T>0$.

Definition 2.1. We say that a nonnegative function $u(x, t) \in C^{2,1}\left(Q_{T}\right) \cap C^{1,0}\left(Q_{T} \cup\right.$ $\left.\Gamma_{T}\right)$ is a supersolution of (1.1)-(1.3) in $Q_{T}$ if

$$
\begin{gathered}
u_{t} \geq \Delta u-c(x, t) u^{p},(x, t) \in Q_{T}, \\
\frac{\partial u(x, t)}{\partial \nu} \geq \int_{\Omega} k(x, y, t) u^{l}(y, t) d y, x \in \partial \Omega, 0 \leq t<T, \\
u(x, 0) \geq u_{0}(x), x \in \Omega,
\end{gathered}
$$

and $u(x, t) \in C^{2,1}\left(Q_{T}\right) \cap C^{1,0}\left(Q_{T} \cup \Gamma_{T}\right)$ is a subsolution of 1.1) (1.3) in $Q_{T}$ if $u \geq 0$ and it satisfies (2.1)-(2.3) in the reverse order. We say that $u(x, t)$ is a solution of problem (1.1)-(1.3) in $Q_{T}$ if $u(x, t)$ is both a subsolution and a supersolution of (1.1) - (1.3) in $Q_{T}$.

Definition 2.2. We say that a solution $u(x, t)$ of (1.1)-(1.3) in $Q_{T}$ is a maximal solution if for any other solution $v(x, t)$ of (1.1) -(1.3) in $Q_{T}$ the inequality $v(x, t) \leq$ $u(x, t)$ is satisfied for $(x, t) \in Q_{T} \cup \Gamma_{T}$.

Definition 2.3. We say that $u$ is a strict supersolution of problem (1.1)-(1.3) in $Q_{T}$ if it is a supersolution in $Q_{T}$ and the inequality in (2.2) is strict. Analogously we say that $u$ is a strict subsolution of problem (1.1) (1.3) in $Q_{T}$ if it is a subsolution in $Q_{T}$ and the inequality in (2.2) is reversed and strict.

Let $\left\{\varepsilon_{m}\right\}$ be decreasing to 0 sequence such that $0<\varepsilon_{m}<1$. For $\varepsilon=\varepsilon_{m}$ let $u_{0 \varepsilon}(x)$ be the functions with the following properties: $u_{0 \varepsilon}(x) \in C^{1}(\bar{\Omega}), u_{0 \varepsilon}(x) \geq \varepsilon$, $u_{0 \varepsilon_{i}}(x) \geq u_{0 \varepsilon_{j}}(x)$ for $\varepsilon_{i}>\varepsilon_{j}, u_{0 \varepsilon}(x) \rightarrow u_{0}(x)$ as $\varepsilon \rightarrow 0$ and

$$
\frac{\partial u_{0 \varepsilon}(x)}{\partial \nu}=\int_{\Omega} k(x, y, 0) u_{0 \varepsilon}^{l}(y) d y
$$

for $x \in \partial \Omega$. Since the nonlinearities in (1.1), (1.2), the Lipschitz condition can be not satisfied, and thus we need to consider the following auxiliary problem:

$$
\begin{cases}u_{t}=\Delta u-c(x, t) u^{p}+c(x, t) \varepsilon^{p} & \text { for } x \in \Omega, t>0 \\ \frac{\partial u(x, t)}{\partial \nu}=\int_{\Omega} k(x, y, t) u^{l}(y, t) d y & \text { for } x \in \partial \Omega, t \geq 0 \\ u(x, 0)=u_{0 \varepsilon}(x) & \text { for } x \in \Omega\end{cases}
$$


where $\varepsilon=\varepsilon_{m}$. The notion of a solution $u_{\varepsilon}$ for problem (2.4) can be defined in a similar way as in the Definition 2.1.

Theorem 2.4. Problem 2.4 has a unique solution in $Q_{T}$ for small values of $T$.

Proof. We start the proof with the construction of a supersolution of (2.4). Let $\sup _{\Omega} u_{0 \varepsilon}(x) \leq M$. Denote $K=\sup _{\partial \Omega \times Q_{1}} k(x, y, t)$ and introduce an auxiliary function $\psi(x)$ with the following properties:

$$
\psi(x) \in C^{2}(\bar{\Omega}), \inf _{\Omega} \psi(x) \geq 1, \inf _{\partial \Omega} \frac{\partial \psi(x)}{\partial \nu} \geq K M^{l-1} \max \{1, \exp (l-1)\} \int_{\Omega} \psi^{l}(y) d y .
$$

Set $\alpha=\sup _{\Omega} \Delta \psi(x)$. Then it is not difficult to check that

$$
w(x, t)=M \exp (\alpha t) \psi(x)
$$

is a supersolution of (2.4) in $Q_{T}$ if $T \leq \min \{1 / \alpha, 1\}$.

To prove the existence of a solution for (2.4) we introduce the set

$$
B=\left\{h(x, t) \in C\left(\overline{Q_{T}}\right): \varepsilon \leq h(x, t) \leq w(x, t), h(x, 0)=u_{0 \varepsilon}(x)\right\}
$$

and consider the following problem

$$
\begin{cases}u_{t}=\Delta u-c(x, t) u^{p}+c(x, t) \varepsilon^{p} & \text { for } x \in \Omega, 0<t<T, \\ \frac{\partial u(x, t)}{\partial \nu}=\int_{\Omega} k(x, y, t) v^{l}(y, t) d y & \text { for } x \in \partial \Omega, 0 \leq t<T, \\ u(x, 0)=u_{0 \varepsilon}(x) & \text { for } x \in \Omega,\end{cases}
$$

where $v \in B$. It is obvious, $B$ is a nonempty convex subset of $C\left(\overline{Q_{T}}\right)$. By classical theory 22] problem (2.5) has a solution $u \in C^{2,1}\left(Q_{T}\right) \cap C^{1,0}\left(\bar{Q}_{T}\right)$. Let us call $A v=u$. In order to show that $A$ has a fixed point in $B$ we verify that $A$ is a continuous mapping from $B$ into itself such that $A B$ is relatively compact. Thanks to a comparison principle for (2.5) we have that $A$ maps $B$ into itself.

Let $G(x, y ; t)$ denote the Green's function for a heat equation given by

$$
u_{t}-\Delta u=0 \text { for } x \in \Omega, t>0
$$

with homogeneous Neumann boundary condition. Then $u(x, t)$ is a solution of (2.5) in $Q_{T}$ if and only if

$$
\begin{aligned}
u(x, t) & =\int_{\Omega} G(x, y ; t) u_{0 \varepsilon}(y) d y+\int_{0}^{t} \int_{\Omega} G(x, y ; t-\tau) c(y, \tau)\left(\varepsilon^{p}-u^{p}(y, \tau)\right) d y d \tau \\
& +\int_{0}^{t} \int_{\partial \Omega} G(x, \xi ; t-\tau) \int_{\Omega} k(\xi, y, \tau) v^{l}(y, \tau) d y d S_{\xi} d \tau
\end{aligned}
$$

for $(x, t) \in Q_{T}$.

We claim that $A$ is continuous. In fact let $v_{k}$ be a sequence in $B$ converging to $v \in B$ in $C\left(\overline{Q_{T}}\right)$. Denote $u_{k}=A v_{k}$. Then by (2.6) we see that

$$
\begin{aligned}
\left|u-u_{k}\right| & \leq \theta \sup _{Q_{T}}\left|u-u_{k}\right| \int_{0}^{t} \int_{\Omega} G(x, y ; t-\tau) c(y, \tau) d y d \tau \\
& +\sup _{Q_{T}}\left|v^{l}-v_{k}^{l}\right| \int_{0}^{t} \int_{\partial \Omega} G(x, \xi ; t-\tau) \int_{\Omega} k(\xi, y, \tau) d y d S_{\xi} d \tau,
\end{aligned}
$$

where $\theta=p \max \left\{\varepsilon^{p-1}, \sup _{Q_{T}} w^{p-1}\right\}$. We note that (see [11])

$$
\theta \sup _{Q_{T}} \int_{0}^{t} \int_{\Omega} G(x, y ; t-\tau) c(y, \tau) d y d \tau<1
$$


for small values of $T$. Now we can conclude that $u_{k}$ converges to $u_{\varepsilon}$ in $C\left(\overline{Q_{T}}\right)$.

The equicontinuity of $A B$ follows from (2.6) and the properties of the Green's function (see, for example, 25]). The Ascoli-Arzelá theorem guarantees the relative compactness of $A B$. Thus we are able to apply the Schauder-Tychonoff fixed point theorem and conclude that $A$ has a fixed point in $B$ if $T$ is small. Now if $u_{\varepsilon}$ is a fixed point of $A, u_{\varepsilon} \in C^{2,1}\left(Q_{T}\right) \cap C^{1,0}\left(\bar{Q}_{T}\right)$ and it is a solution of (2.4) in $Q_{T}$. Uniqueness of the solution follows from a comparison principle for (2.4) which can be proved in a similar way as in the next section.

Now, let $\varepsilon_{2}>\varepsilon_{1}$. Then it is easy to see that $u_{\varepsilon_{2}}(x, t)$ is a supersolution of problem (2.4) with $\varepsilon=\varepsilon_{1}$. Applying to problem (2.4) a comparison principle we have $u_{\varepsilon_{1}}(x, t) \leq u_{\varepsilon_{2}}(x, t)$. Using the last inequality and the continuation principle of solutions we deduce that the existence time of $u_{\varepsilon}$ does not decrease as $\varepsilon \searrow 0$. Taking $\varepsilon \rightarrow 0$, we get $u_{m}(x, t)=\lim _{\varepsilon \rightarrow 0} u_{\varepsilon}(x, t) \geq 0$ and $u_{m}(x, t)$ exists in $Q_{T}$ for some $T>0$. By dominated convergence theorem, $u_{m}(x, t)$ satisfies the following equation

$$
\begin{aligned}
u_{m}(x, t) & \left.=\int_{\Omega} G(x, y ; t) u_{0}(y) d y-\int_{0}^{t} \int_{\Omega} G(x, y ; t-\tau) c(y, \tau) u_{m}^{p}(y, \tau)\right) d y d \tau \\
& +\int_{0}^{t} \int_{\partial \Omega} G(x, \xi ; t-\tau) \int_{\Omega} k(\xi, y, \tau) u_{m}^{l}(y, \tau) d y d \xi d \tau
\end{aligned}
$$

for $(x, t) \in Q_{T}$. Further, the interior regularity of $u_{m}(x, t)$ follows from the continuity of $u_{m}(x, t)$ in $Q_{T}$ and the properties of the Green's function. Obviously, $u_{m}(x, t)$ satisfies (1.1)-(1.3).

Theorem 2.5. Problem (1.1)-(1.3) has a solution in $Q_{T}$ for small values of $T$.

\section{UNIQUENESS AND NONUNIQUENESS}

We start this section with a comparison principle for problem (1.1)-1.3 which is used below.

Theorem 3.1. Let $\bar{u}$ and $\underline{u}$ be a supersolution and a subsolution of problem (1.1)(1.3) in $Q_{T}$, respectively. Suppose that $\underline{u}(x, t)>0$ or $\bar{u}(x, t)>0$ in $Q_{T} \cup \Gamma_{T}$ if $l<1$. Then $\bar{u}(x, t) \geq \underline{u}(x, t)$ in $Q_{T} \cup \Gamma_{T}$.

Proof. Suppose that $l \geq 1$. Let $T_{0} \in(0, T)$ and $u_{0 \varepsilon}(x)$ have the same properties as in previous section but $u_{0 \varepsilon}(x) \rightarrow \underline{u}(x, 0)$ as $\varepsilon \rightarrow 0$. We can construct a solution $u_{m}(x, t)$ of (1.1)-(1.3) with $u_{0}(x)=\underline{u}(x, 0)$ in the following way $u_{m}(x, t)=\lim _{\varepsilon \rightarrow 0} u_{\varepsilon}(x, t)$ where $u_{\varepsilon}(x, t)$ is a solution of (2.4). To establish theorem we shall show that

$$
\underline{u}(x, t) \leq u_{m}(x, t) \leq \bar{u}(x, t) \text { in } \bar{Q}_{T_{0}} \text { for any } T_{0} .
$$

We prove the second inequality in (3.1) only since the proof of the first one is similar. Let $\varphi(x, t) \in C^{2,1}\left(\bar{Q}_{T_{0}}\right)$ be a nonnegative function such that

$$
\frac{\partial \varphi(x, t)}{\partial \nu}=0,(x, t) \in S_{T_{0}} .
$$

If we multiply the first equation in (2.4) by $\varphi(x, t)$ and then integrate over $Q_{t}$ for $0<t<T_{0}$, we get

$$
\int_{\Omega} u_{\varepsilon}(x, t) \varphi(x, t) d x \leq \int_{\Omega} u_{\varepsilon}(x, 0) \varphi(x, 0) d x+\varepsilon^{p} \int_{0}^{t} \int_{\Omega} c(x, \tau) \varphi d x d \tau
$$




$$
\begin{aligned}
& +\int_{0}^{t} \int_{\Omega}\left(u_{\varepsilon} \varphi_{\tau}+u_{\varepsilon} \Delta \varphi-c(x, \tau) u_{\varepsilon}^{p} \varphi\right) d x d \tau \\
& +\int_{0}^{t} \int_{\partial \Omega} \varphi(x, \tau) \int_{\Omega} k(x, y, \tau) u_{\varepsilon}^{l}(y, \tau) d y d S_{x} d \tau
\end{aligned}
$$

On the other hand, $\bar{u}$ satisfies (3.2) with reversed inequality and with $\varepsilon=0$. Set $w(x, t)=u_{\varepsilon}(x, t)-\bar{u}(x, t)$. Then $w(x, t)$ satisfies

$$
\begin{aligned}
\int_{\Omega} w(x, t) \varphi(x, t) d x & \leq \int_{\Omega} w(x, 0) \varphi(x, 0) d x+\varepsilon^{p} \int_{0}^{t} \int_{\Omega} c(x, \tau) \varphi d x d \tau \\
& \left.+\int_{0}^{t} \int_{\Omega}\left(\varphi_{\tau}+\Delta \varphi-c(x, \tau) p \theta_{1}^{p-1} \varphi\right)\right) w d x d \tau \\
& +\int_{0}^{t} \int_{\partial \Omega} \varphi(x, \tau) \int_{\Omega} k(x, y, \tau) l \theta_{2}^{l-1} w(y, \tau) d y d S_{x} d \tau
\end{aligned}
$$

where $\theta_{1}$ and $\theta_{2}$ are some continuous functions between $u_{\varepsilon}$ and $\bar{u}$. Note here that by hypotheses for $c(x, t), k(x, y, t), u_{\varepsilon}(x, t)$ and $\bar{u}(x, t)$, we have

$$
\begin{aligned}
& 0 \leq c(x, t) \leq M, 0 \leq \bar{u}(x, t) \leq M \varepsilon \leq u_{\varepsilon}(x, t) \leq M \text { in } \bar{Q}_{T_{0}} \\
& \text { and } 0 \leq k(x, y, t) \leq M \text { in } \partial \Omega \times \bar{Q}_{T_{0}},
\end{aligned}
$$

where $M$ is some positive constant. Then, it is easy to see from (3.4) that $\theta_{1}^{p-1}$ and $\theta_{2}^{l-1}$ are positive and bounded functions in $\bar{Q}_{T_{0}}$ and moreover, $\theta_{2}^{l-1} \leq M^{l-1}$. Define a sequence $\left\{a_{n}\right\}$ in the following way: $a_{n} \in C^{\infty}\left(\bar{Q}_{T_{0}}\right), a_{n} \geq 0$ and $a_{n} \rightarrow$ $c(x, t) p \theta_{1}^{p-1}$ as $n \rightarrow \infty$ in $L^{1}\left(Q_{T_{0}}\right)$. Now, consider a backward problem given by

$$
\begin{cases}\varphi_{\tau}+\Delta \varphi-a_{n} \varphi=0 & \text { for } x \in \Omega, 0<\tau<t \\ \frac{\partial \varphi(x, \tau)}{\partial \nu}=0 & \text { for } x \in \partial \Omega, 0 \leq \tau<t \\ \varphi(x, t)=\psi(x) & \text { for } x \in \Omega,\end{cases}
$$

where $\psi(x) \in C_{0}^{\infty}(\Omega)$ and $0 \leq \psi(x) \leq 1$. Denote a solution of (3.5) as $\varphi_{n}(x, \tau)$. Then by the standard theory for linear parabolic equations (see [22], for example), we find that $\varphi_{n} \in C^{2,1}\left(\bar{Q}_{t}\right), 0 \leq \varphi_{n}(x, \tau) \leq 1$ in $\bar{Q}_{t}$. Putting $\varphi=\varphi_{n}$ in (3.3) and passing then to the limit as $n \rightarrow \infty$ we infer

$$
\int_{\Omega} w(x, t) \psi(x) d x \leq \int_{\Omega} w(x, 0)_{+} d x+\varepsilon^{p} M T_{0}|\Omega|+l M^{l}|\partial \Omega| \int_{0}^{t} \int_{\Omega} w(y, \tau)_{+} d y d \tau,
$$

where $w_{+}=\max \{w, 0\},|\partial \Omega|$ and $|\Omega|$ are the Lebesgue measures of $\partial \Omega$ in $\mathbb{R}^{n-1}$ and $\Omega$ in $\mathbb{R}^{n}$, respectively. Since (3.6) holds for every $\psi(x)$, we can choose a sequence $\left\{\psi_{n}\right\}$ converging on $\Omega$ to $\psi(x)=1$ if $w(x, t)>0$ and $\psi(x)=0$ otherwise. Hence, from (3.6) we get

$$
\int_{\Omega} w(x, t)_{+} d x \leq \int_{\Omega} w(x, 0)_{+} d x+\varepsilon^{p} M T_{0}|\Omega|+l M^{l}|\partial \Omega| \int_{0}^{t} \int_{\Omega} w(y, \tau)_{+} d y d \tau .
$$

Applying now Gronwall's inequality and passing to the limit $\varepsilon \rightarrow 0$, the conclusion of this theorem follows for $l \geq 1$. For the case $l<1$ we can consider $w(x, t)=$ $\underline{u}(x, t)-\bar{u}(x, t)$ and prove the theorem in a similar way using the positiveness of a subsolution or a supersolution.

Corollary 3.2. Problem (1.1)-(1.3) has a maximal solution in $Q_{T}$ for small values of $T$. 
Proof. In the previous section we prove the existence of a local solution $u_{m}(x, t)=$ $\lim _{\varepsilon \rightarrow 0} u_{\varepsilon}(x, t)$. Let $v(x, t)$ be any other solution of (1.1)-(1.3) in $Q_{T}$. By Theorem 3.1 we have $u_{\varepsilon}(x, t) \geq v(x, t)$. Taking $\varepsilon \rightarrow 0$, we conclude $u_{m}(x, t) \geq v(x, t)$. Obviously, $u_{m}(x, t)$ is a maximal solution of (1.1) (1.3) in $Q_{T}$.

Next lemma shows the positiveness of all nontrivial solutions for $t>0$ if $p \geq 1$.

Lemma 3.3. Let $u_{0}$ is a nontrivial function in $\Omega, p \geq 1$ or $c(x, t) \equiv 0$. Suppose $u$ is a solution of (1.1)-(1.3) in $Q_{T}$. Then $u>0$ in $Q_{T} \cup S_{T}$.

Proof. As $c(x, t)$ and $u(x, t)$ are continuous in $\bar{Q}_{T}$ functions then we have

$$
\max \{c(x, t), u(x, t)\} \leq M,(x, t) \in \bar{Q}_{T}
$$

with some positive constant $M$. Now we put $v=u \exp (\lambda t)$ where $\lambda \geq M^{p}$. It is easy to verify that $v_{t}-\Delta v \geq 0$. Since $v(x, 0)=u_{0}(x) \not \equiv 0$ in $\Omega$ and $v(x, t) \geq 0$ in $Q_{T}$, by the strong maximum principle $v(x, t)>0$ in $Q_{T}$. Let $v\left(x_{0}, t_{0}\right)=0$ in some point $\left(x_{0}, t_{0}\right) \in S_{T}$. Then according to Theorem 3.6 of [10] it yields $\partial v\left(x_{0}, t_{0}\right) / \partial \nu<0$, which contradicts (1.2).

As a simple consequence of Theorem 3.1 and Lemma 3.3 , we get the following uniqueness result for problem (1.1)-(1.3)

Theorem 3.4. Let problem (1.1)-(1.3) has a solution in $Q_{T}$ with nonnegative initial data for $l \geq 1$ and with positive initial data under the conditions $l<1, p \geq 1$ or a positive in $Q_{T} \cup \Gamma_{T}$ solution if $\max (p, l)<1$. Then a solution of (1.1)-(1.3) is unique in $Q_{T}$.

Now we shall prove the nonuniqueness of a solution of our problem with trivial initial datum for $l<p$. We note that problem (1.1)-(1.3) with trivial initial datum has trivial solution.

Theorem 3.5. Let $l<\min \{1, p\}$ and $u_{0}(x) \equiv 0$. Suppose that

$$
k\left(x, y_{0}, t_{0}\right)>0 \text { for any } x \in \partial \Omega \text { and some } y_{0} \in \partial \Omega \text { and } t_{0} \in[0, T) .
$$

Then maximal solution $u_{m}(x, t)$ of problem (1.1)-(1.3) is nontrivial function in $Q_{T}$.

Proof. As we showed in Theorem 2.5 and Corollary 3.2 a maximal solution $u_{m}(x, t)=$ $\lim _{\varepsilon \rightarrow 0} u_{\varepsilon}(x, t)$, where $u_{\varepsilon}(x, t)$ is some positive in $\bar{Q}_{T}$ supersolution of (1.1) $-(1.3)$. To prove theorem we construct a nontrivial nonnegative subsolution $\underline{u}(x, t)$ of (1.1) (1.3) with trivial initial datum. By Theorem 3.1 then we have $u_{\varepsilon}(x, t) \geq \underline{u}(x, t)$ and therefore maximal solution $u_{m}(x, t)$ is nontrivial function.

To construct a subsolution we use the change of variables in a neighborhood of $\partial \Omega$ as in $\underline{3}$. Let $\bar{x}$ be a point in $\partial \Omega$. We denote by $\widehat{n}(\bar{x})$ the inner unit normal to $\partial \Omega$ at the point $\bar{x}$. Since $\partial \Omega$ is smooth it is well known that there exists $\delta>0$ such that the mapping $\psi: \partial \Omega \times[0, \delta] \rightarrow \mathbb{R}^{n}$ given by $\psi(\bar{x}, s)=\bar{x}+s \widehat{n}(\bar{x})$ defines new coordinates $(\bar{x}, s)$ in a neighborhood of $\partial \Omega$ in $\bar{\Omega}$.

A straightforward computation shows that, in these coordinates, $\Delta$ applied to a function $g(\bar{x}, s)=g(s)$, which is independent of the variable $\bar{x}$, evaluated at a point $(\bar{x}, s)$ is given by

$$
\Delta g(\bar{x}, s)=\frac{\partial^{2} g}{\partial s^{2}}(\bar{x}, s)-\sum_{j=1}^{n-1} \frac{H_{j}(\bar{x})}{1-s H_{j}(\bar{x})} \frac{\partial g}{\partial s}(\bar{x}, s),
$$


where $H_{j}(\bar{x})$ for $j=1, \ldots, n-1$, denotes the principal curvatures of $\partial \Omega$ at $\bar{x}$.

Under the assumptions of the theorem there exists $\bar{t}>0$ such that $k(x, y, t)>0$ for $t_{0} \leq t \leq t_{0}+\bar{t}, x \in \partial \Omega$ and $y \in V\left(y_{0}\right)$, where $V\left(y_{0}\right)$ is some neighborhood of $y_{0}$ in $\bar{\Omega}$.

Let $1 /(1-l)<\alpha \leq 1 /(1-p)$ for $p<1$ and $\alpha>1 /(1-l)$ for $p \geq 1,2<\beta<$ $2 /(1-p)$ for $p<1$ and $\beta>2$ for $p \geq 1$ and assume that $A>0,0<\xi_{0} \leq 1$ and $0<T_{0}<\min \left(T-t_{0}, \bar{t}, \delta^{2}\right)$. For points in $\partial \Omega \times[0, \delta] \times\left(t_{0}, t_{0}+T_{0}\right]$ of coordinates $(\bar{x}, s, t)$ define

$$
\underline{u}(\bar{x}, s, t)=A\left(t-t_{0}\right)^{\alpha}\left(\xi_{0}-\frac{s}{\sqrt{t-t_{0}}}\right)_{+}^{\beta}
$$

and extend $\underline{u}$ as zero to the whole of $\overline{Q_{\tau}}$ with $\tau=t_{0}+T_{0}$. Using (3.9), we get that

$$
\begin{aligned}
& \underline{u}_{t}(\bar{x}, s, t)-\Delta \underline{u}(\bar{x}, s, t)+c(x, t) \underline{u}^{p}(\bar{x}, s, t)=\alpha A\left(t-t_{0}\right)^{\alpha-1}\left(\xi_{0}-\frac{s}{\sqrt{t-t_{0}}}\right)_{+}^{\beta} \\
& +\frac{\beta}{2} A s\left(t-t_{0}\right)^{\alpha-3 / 2}\left(\xi_{0}-\frac{s}{\sqrt{t-t_{0}}}\right)_{+}^{\beta-1}-\beta(\beta-1) A\left(t-t_{0}\right)^{\alpha-1}\left(\xi_{0}-\frac{s}{\sqrt{t-t_{0}}}\right)_{+}^{\beta-2} \\
& -\beta A\left(t-t_{0}\right)^{\alpha-1 / 2}\left(\xi_{0}-\frac{s}{\sqrt{t-t_{0}}}\right)_{+}^{\beta-1} \sum_{j=1}^{n-1} \frac{H_{j}(\bar{x})}{1-s H_{j}(\bar{x})} \\
& +A^{p} c(x, t)\left(t-t_{0}\right)^{\alpha p}\left(\xi_{0}-\frac{s}{\sqrt{t-t_{0}}}\right)_{+}^{\beta p} \leq 0
\end{aligned}
$$

for $(\bar{x}, s, t) \in \partial \Omega \times(0, \delta] \times\left(t_{0}, \tau\right)$ and small values of $\xi_{0}$.

It is obvious,

$$
\frac{\partial \underline{u}}{\partial \nu}(\bar{x}, 0, t)=-\frac{\partial \underline{u}}{\partial s}(\bar{x}, 0, t)=\beta A\left(t-t_{0}\right)^{\alpha-\frac{1}{2}} \xi_{0}^{\beta-1}, \bar{x} \in \partial \Omega, t_{0}<t<\tau .
$$

To prove that $\underline{u}$ is the subsolution of (1.1)-(1.3) in $Q_{\tau}$ it is enough to check the validity of the following inequality

$\beta A\left(t-t_{0}\right)^{\alpha-\frac{1}{2}} \xi_{0}^{\beta-1} \leq A^{l}\left(t-t_{0}\right)^{\alpha l} \int_{\partial \Omega \times[0, \delta]} k(x,(\bar{y}, s), t)|J(\bar{y}, s)|\left(\xi_{0}-\frac{s}{\sqrt{t-t_{0}}}\right)_{+}^{\beta l} d \bar{y} d s$

for $x \in \partial \Omega$ and $t_{0}<t<\tau$. Here $J(\bar{y}, s)$ is Jacobian of the change of variables. Estimating the integral $I$ in the right-hand side of (3.11)

$I=\left(t-t_{0}\right)^{\frac{1}{2}} \int_{\partial \Omega} d \bar{y} \int_{0}^{\xi_{0}} k\left(x,\left(\bar{y}, z \sqrt{t-t_{0}}\right), t\right)\left|J\left(\bar{y}, z \sqrt{t-t_{0}}\right)\right|\left(\xi_{0}-z\right)_{+}^{\beta l} d z \geq C\left(t-t_{0}\right)^{\frac{1}{2}}$,

where positive constant $C$ does not depend on $t$, we obtain that (3.11) is true if we take $T_{0}$ sufficiently small.

Remark 3.6. Let $c\left(x, t_{0}\right) \leq c_{0}$ and $k\left(x, y, t_{0}\right) \geq k_{0}$ for $x \in \Omega, y \in \partial \Omega$, some $t_{0} \in[0, T)$ and positive constants $c_{0}$ and $k_{0}$. Then nonuniqueness of trivial solution for our problem holds for $l=p<1$ and large values of $k_{0} / c_{0}$. To prove this we can take in (3.10) $\alpha=1 /(1-l), \beta=2 /(1-l)$ and $A$ in a suitable way.

To prove the uniqueness of trivial solution of (1.1)-(1.3) with $u_{0}(x) \equiv 0$ we need the following comparison principle. 
Lemma 3.7. Let $\underline{u}$ and $\bar{u}$ be a subsolution and a strict supersolution of (1.1)-(1.3) in $Q_{T}$, respectively. Then $\bar{u} \geq \underline{u}$ in $\bar{Q}_{T}$.

Proof. Let $v=\bar{u}+\varepsilon$ for $\varepsilon>0$. Note that for any $T_{0}<T$ under suitable choice of $\varepsilon$ we have

$$
\frac{\partial v(x, t)}{\partial \nu} \geq \int_{\Omega} k(x, y, t) v^{l}(y, t) d y \text { for } x \in \partial \Omega, 0 \leq t \leq T_{0} .
$$

Now we can apply Theorem 3.1 to get the inequality $v \geq \underline{u}$ in $\bar{Q}_{T_{0}}$. Passing here to the limit as $\varepsilon \rightarrow 0$ we prove the lemma.

Theorem 3.8. Let $p<l<1$ and $u_{0}(x) \equiv 0$. Suppose that $c(x, t) \geq c_{1}>0$ for $(x, t) \in \bar{Q}_{T}$. Then the solution $u \equiv 0$ of problem (1.1)-(1.3) is unique in $Q_{T}$.

Proof. Since there is the comparison principle for a solution and a strict supersolution of (1.1)-(1.3), it is sufficiently to construct arbitrarily small strict supersolutions which have a positive values on $\partial \Omega$ (see [5] for another problem).

We shall use the change of variables in a neighborhood of $\partial \Omega$ which we introduced in Theorem 3.5. Let $(1-l) / 2<\gamma<(1-p) / 2,0<\varepsilon<\delta^{1 / \gamma}, A>0$ and $0<\xi_{0} \leq 1$. For points in $\partial \Omega \times[0, \delta] \times[0, T]$ of coordinates $(\bar{x}, s, t)$ define

$$
\bar{u}_{\varepsilon}(\bar{x}, s, t)=\varepsilon A\left(\xi_{0}-\varepsilon^{-\gamma} s\right)_{+}^{1 / \gamma}
$$

and extend $\underline{u}_{\varepsilon}$ as zero to the whole of $\overline{Q_{T}}$. Using (3.9), we get that

$$
\bar{u}_{\varepsilon t}(\bar{x}, s, t)-\Delta \bar{u}_{\varepsilon}(\bar{x}, s, t)+c(x, t) \bar{u}_{\varepsilon}^{p}(\bar{x}, s, t) \geq 0
$$

in $Q_{T}$ for small values of $\xi_{0}$.

To show that $\bar{u}_{\varepsilon}$ is a strict supersolution we need to prove the following inequality

$$
\frac{A}{\gamma} \varepsilon^{1-\gamma} \xi_{0}^{(1-\gamma) / \gamma}>(\varepsilon A)^{l} \int_{\partial \Omega \times[0, \delta]} k(x,(\bar{y}, s), t)|J(\bar{y}, s)|\left(\xi_{0}-\varepsilon^{-\gamma} s\right)_{+}^{l / \gamma} d \bar{y} d s
$$

for $x \in \partial \Omega$ and $0 \leq t<T$. Let $k(x, y, t) \leq k_{1}$ in $Q_{T}$. Then estimating the right-hand side of (3.13) $J$, we get

$$
J \leq k_{1} C A^{l} \varepsilon^{l+\gamma} \xi_{0}^{(l+\gamma) / \gamma},
$$

where positive constant $C$ depend only on $p, l$ and $\partial \Omega$. Hence (3.13) holds if we take $\xi_{0}$ small enough.

We are in a position to complete the proof. Suppose for a contradiction that there exists a solution of (1.1) (1.3) with trivial initial function which is not identically zero in $Q_{T}$. Then by Lemma 3.7 it follows that

$$
u(x, t) \leq u_{\varepsilon}(x, t) \text { in } Q_{T}
$$

for all $0<\varepsilon<\delta^{1 / \gamma}$. This is a contradiction since $u_{\varepsilon} \rightarrow 0$ uniformly on $\bar{Q}_{T}$ as $\varepsilon \rightarrow 0$.

Remark 3.9. Let the assumptions of Theorem 3.8 fulfill but only $l=p$. Then the conclusion of Theorem 3.8 holds for large values of $c_{1} / k_{1}$, where $k_{1}$ was defined in the proof. To prove this we can take in (3.12) $\gamma=(1-l) / 2$ and $A$ in a suitable way. 
Remark 3.10. Note that under the assumptions of Theorem 3.8 or Remark 3.9 there exists a class of nontrivial initial data such that $u(x, t) \equiv 0$ for large values of $t$. To prove this we can modify function $\bar{u}_{\varepsilon}$ from (3.12) in the following way

$$
\bar{u}_{\varepsilon}(\bar{x}, s, t)=\varepsilon\left(\xi_{0}-\varepsilon^{-\gamma} s-\mu t\right)_{+}^{1 / \gamma}, \mu>0 .
$$

\section{REFERENCES}

[1] J. Aguirre and M.Escobedo, A Cauchy problem for $u_{t}-\Delta u=u^{p}$ : Asymptotic behavior of solutions, Ann. Fac. Sci. Toulouse 8 (1986-87), 175-203.

[2] P. Bokes, A uniqueness result for a semilinear parabolic system, J. Math. Anal. Appl. 331 (2007), 567-584.

[3] C. Cortazar, M. del Pino and M. Elgueta, On the short-time behaviour of the free boundary of a porous medium equation, Duke Math. J. 87 (1997), no. 1, 133-149.

[4] C. Cortazar, M. Elgueta and J. D. Rossi, Uniqueness and non-uniqueness for a system of heat equations with non-linear coupling at the boundary, Nonlinear Anal. 37 (1999), no. 2, 257-267.

[5] C. Cortazar, M. Elgueta and J. D. Rossi, Uniqueness and nonuniqueness for the porous medium equation with non linear boundary condition, Diff. Int. Equat. 16 (2003), no. 10, $1215-1222$.

[6] K. Deng, Comparison principle for some nonlocal problems, Quart. Appl. Math. 50 (1992), no. $3,517-522$.

[7] M. Escobedo and M. A. Herrero, A semilinear parabolic system in a bounded domain, Ann. Mat. Pura Appl. CLXV (1993), 315-336.

[8] Z.B. Fang and J. Zhang, Global and blow-up solutions for the nonlocal p-Laplacian evolution equationwith weighted nonlinear nonlocal boundary condition, J. Integral Equat. Appl. 26 (2014), no. 2, 171-196.

[9] H. Fujita and S. Watanabe, On the uniqueness and non-uniqueness of solutions of initial value problems for some quasi-linear parabolic equations, Comm. Pure Appl. Math. 21 (1968), no. 6, 631-652.

[10] B. Hu, Blow-up theories for semilinear parabolic equations, Lecture Notes in Mathematics 2018 (2011), 127 p.

[11] B. Hu and H.-M. Yin, Critical exponents for a system of heat equations coupled in a nonlinear boundary condition, Math. Methods Appl. Sci. 19 (1996), no. 14, 1099-1120.

[12] Y. Gao and W. Gao, Existence and blow-up of solutions for a porous medium equation with nonlocal boundary condition, Appl. Anal. 90 (2011), no. 5, 799-809.

[13] A. Gladkov and M. Guedda, Blow-up problem for semilinear heat equation with absorption and a nonlocal boundary condition, Nonlinear Anal. 74 (2011), no. 13, 4573-4580.

[14] A. Gladkov and M. Guedda, Semilinear heat equation with absorption and a nonlocal boundary condition, Appl. Anal. 91 (2012), no. 12, 2267-2276.

[15] A. Gladkov and T. Kavitova, Initial boundary value problem for a semilinear parabolic equation with nonlinear nonlocal boundary conditions, Ukrain. Math. J., article in press.

[16] A. Gladkov and T. Kavitova, Blow-up problem for semilinear heat equation with nonlinear nonlocal boundary condition, Appl. Anal., article in press.

[17] A. Gladkov and K. I. Kim, Blow-up of solutions for semilinear heat equation with nonlinear nonlocal boundary condition, J. Math. Anal. Appl. 338 (2008), 264-273.

[18] A. Gladkov and K. I. Kim, Uniqueness and nonuniqueness for reaction-diffusion equation with nonlocal boundary condition, Adv. Math. Sci. Appl. 19 (2009), no. 1, 39-49.

[19] A. Gladkov and A. Nikitin, A reaction-diffusion system with nonlinear nonlocal boundary conditions, Int. J. Partial Differential Equations 2014 (2014), Article ID 523656, 10 pages.

[20] C. S. Kahane, On the asymptotic behavior of solutions of parabolic equations, Czechoslovak Math. J. 33 (1983), no. 108, 262-285.

[21] M. Kordos̆, On uniqueness for a semilinear parabolic system coupled in an equation and a boundary condition, J. Math. Anal. Appl. 298 (2004), 655-666.

[22] Ladyzhenskaja, O., Solonnikov, V. and Ural'ceva, N., Linear and quasilinear equations of parabolic type, Transl. Math. Monographs 23(1968).

[23] D. Liu, Blow-up for a degenerate and singular parabolic equation with nonlocal boundary condition, J. Nonlinear Sci. Appl. 9 (2016), 208-218. 
[24] D. Liu and C. Mu, Blowup properties for a semilinear reaction-diffusion system with nonlinear nonlocal boundary conditions, Abstr. Appl. Anal. 2010 (2010), Article ID 148035, 17 pages.

[25] C. V. Pao, Nonlinear parabolic and elliptic equations, Plenum Press, New York, 1992.

[26] G. Zhong and L. Tian Blow up problems for a degenerate parabolic equation with nonlocal source and nonlocal nonlinear boundary condition, Boundary Value Problems 2012 (2012), no. 45, 14 pages.

[27] J. Zhou and D. Yang Blowup for a degenerate and singular parabolic equation with nonlocal source and nonlocal boundary, Appl. Math. Comput. 256 (2015), 881884.

Alexander Gladkov, Department of Mechanics and Mathematics, Belarusian State University, Nezavisimosti Avenue 4, 220030 Minsk, Belarus

E-mail address: gladkoval@mail.ru 